Can these divergent views of the contribution of $\mathrm{HBV}$ to oncogenesis be reconciled? There is no reason why both direct and indirect mechanisms cannot operate in vivo. The fact that liver injury of any cause invariably provokes hepatocyte proliferation means that even in the absence of a specific genetic contribution from the viral genome, there is likely to be a background of transforming events not directly linked to viral DNA. For proponents of direct models, the challenge now is to define to what extent subsets of human $\mathrm{HCC}$ exist in which integrated $\mathrm{HBV}$ sequences act to drive proliferation over and above this background, and to clarify the mechanisms by which this occurs. Clues to such cases might emerge from consideration of the differences between WHV- and HBV- associated oncogenesis, chief among which is the earlier onset of hepatoma during chronic WHV infection. Perhaps the subset of human HCCs that develop earlier than usual in the carrier state would be enriched for such integrants. The finding of frequent oncogene activation in woodchuck HCC will surely invigorate the search for comparable events in human hepatomas.

Don Ganem is in the Departments of Microbiology and Immunology, and Medicine, University of California, San Francisco, California 94143, USA.

\footnotetext{
1. Fourel, G. et al. Nature 347, 294-298 (1990).

2. Beasley, R. P., Lin, C. C., Hwang, L. L.-Y. \& Chen, C.-S Lancet 2, 1129-1133 (1981).

3. Popper, H., Roth, L., Purcell, R., Tennant, B. \& Gerin, J. Proc. natn. Acad. Sci. U.S.A. 84, 866-870 (1987).

4. Dejean, A., Bougeleret, L., Grzeschik, K. \& Tioliais, P. Nature 322, 70-72 (1986).

5. Wang, J., Chenivesse, X., Henglein, B. \& Brechot, C. Nature 343, 555-557 (1990).

6. Hsu, T. et al. Cell 55, 627-635 (1988).

7. Schwab, M. et al. Nature 305, 245-248 (1983)

8. van Lohuiozen, M., Breuer, M. \& Berns, A. EMBO J. 8. 133-136 (1989)

9. Hino, O., Shows, T. \& Rogler, C.E. Proc. natn. Acad. Sci. U.S.A. 83, 8338-8342 (1986).

10. Takada, S. \& Koike, K. Proc. natn. Acad. Sci. U. S. A. 87 5628-5632 (1990).

11. Kekule, A. et al. Nature 343, 457-461 (1990)

12. Chisari, F.V. et al. Cell 59, 1145-1156 (1989).
}

\section{Enhancing mantle conductivity}

\section{M. Hirsch}

THE cause of a layer of high electrical conductivity in the Earth's upper mantle at depths in the region of $40-180 \mathrm{~km}$ has long intrigued and puzzled geophysicists. Both above and below the layer, conductivity profiles can be reconciled with laboratory conductivities of mantle minerals under controlled conditions ${ }^{2.3}$. Conversely, conductivities in the highconductivity layer are about 1-2 orders of magnitude greater than would be reasonably expected from solid-state conduction in the volumetrically most important minerals. Small amounts of good conductors such as partial melt ${ }^{4}$, water ${ }^{5}$ or carbon ${ }^{2}$ are often invoked to explain this anomaly. The common approach is to treat the layer as a mixed medium containing insulating silicate crystals mixed with an interconnected fluid or grain-boundary phase of high conductivity. On page 272 of this issue ${ }^{6}$, Karato presents a new possibility: hydrogen dissolved in the olivine lattice may substantially increase the electrical conductivity of the host crystal. Thus, enhanced conduction outside mineral grains would not be necessary.

Karato bases his idea on diffusivity measurements of hydrogen?, assuming that the hydrogen contributes as charged ions, presumably protons, to electrical conduction in olivine. Because hydrogen is mobile in the [100] crystallographic direstion of olivine, only a modest amount of hydrogen - $\mathrm{H} / \mathrm{Si}$ content between about 200-2,000 parts per million would be sufficient to account for the high-conductivity layer. Although direct evidence of such hydrogen concentrations within olivines and their fluid inclusions from most mantle-derived xenoliths is limited ${ }^{8}$, this circumstance may simply be caused by hydrogen loss during ascent because of its rapid diffusivity in olivine'

Many mechanical and petrological stability problems are avoided with Karato's hydrogen hypothesis because the hydrogen is intracrystalline and the amount of hydrogen required to enhance conductivity is below that which would cause extensive partial melting ${ }^{6}$. In contrast, with the water or melt hypotheses, one is left with the problem of explaining how the mechanical stability of an interconnected fluid phase can be maintained over long distances in the upper mantle for long geological times ${ }^{+}$. And the carbon explanation is constrained by the temperature and pressure for diamond stability, the grain-boundary diffusivity of carbon, and oxidation state of the upper mantle ${ }^{2}$.

To verify Karato's hypothesis, several issues need to be resolved. First, it must be experimentally demonstrated that hydrogen exists as a charged defect in olivine at high temperatures and pressures. Second, we need estimates of the hydrogen content of the upper mantle and the relative partitioning of hydrogen among co-existing mantle minerals. Third, we require a chemical rationale that explains why enhanced conductivity by hydrogen does not persist beyond the high conductivity layer at both shallower and deeper depths. Finally, we need to evaluate what influence the considerable anisotropy of hydrogen diffusivity in oli- vine, spanning two orders of magnitude ${ }^{7}$, may have on the amount of hydrogen required for enhanced conduction. For isotropic mixtures, this anisotropy would increase by a factor of only about 3 Karato's values for the hydrogen content. But significant texture of olivine in the region of the high-conductivity layer could produce a more profound effect on hydrogenenhanced electrical conductivity.

The cause of the high-conductivity layer is a critical question in geophysics. This layer is located in a region of the upper mantle that has several remarkable features that are intimately connected with the thermal and rheological state of the Earth's mantle and crust. Beneath the rigid lithospheric plates $(70 \mathrm{~km}$ thick on average) there is the asthenosphere, a more plastic layer that permits vertical isostatic adjustments of land masses and lateral shear to occur. This shear is driven by convective motions in the mantle and has a direct bearing on the motion of the plates and surface deformations. Another feature is the low-velocity zone - an effective decrease in seismic-wave velocity that starts within a depth range of about 50 to $220 \mathrm{~km}$. Many geophysicists argue that partial melting gives a comprehensive explanation for the low-velocity zone, high-conductivity layer and the weakness of the asthenosphere. The mechanisms responsible for each of these features, however, could differ: both the high-conductivity layer and the lowvelocity zone are often absent beneath continental shields whereas isostatic adjustments from the last ice age, such as in the Canadian and Baltic shields, indicate the presence of the asthenosphere. Yet, although there is no necessary connection between high conductivity and asthenospheric plasticity, hydrogen concentrations of the order $100-3,000$ p.p.m., such as invoked by Karato, may also weaken the upper mantle by a factor of $1.5-3$ (refs 9,10 ).

Of course, high electrical conductivity can have many causes. We may find that high conductivity layers in different localities in fact have different origins.

L. M. Hirsch is in the Geophysics Group and the Institute of Geophysics and Planetary Physics, Los Alamos National Laboratory, Los Alamos, New Mexico 87545, USA.

1. Oldenburg, D. W., Whitall, K. P. \& Parker, R.L. J. geophys. Res. 89, 1829-1833 (1984).

2. Duba, A. G. \& Shankland, T. J. Geophys. Res. Lett. 9 1271-1274 (1982).

3. Shankland, T. J.\& Duba, A. G. Geophys, JIR. astr. Soc. (in the press

4. Shankland, T. J., O'Connell, R. J. \& Waff, H. S. Rev. geophys. Space Phys. 19, 394-406 (1981)

5. Tozer, D. C. Phys. Earth planet. Interiors 25, 280 (1981).

6. Karato, S. Nature 347, 272-273 (1990).

7. Mackwell, S. J. \& Kohlstedt, D. L. J. geophys. Res. 95 5079-5088 (1990).

8. Arai, S. \& Hirai, H. Nature 318, 276-277 (1985).

9. Mackwell, S. J., Kohlstedt, D. L. \& Paterson, M. S. geophys. Res. 90, 11319-11333 (1985).

10. Karato, S. L., Paterson, M. S. \& FitzGerald, J. D. J. geophys. Res. 91, 8151-8176 (1986). 